\title{
Automotive Start-Stop Engine Based on Fingerprint Recognition System
}

\author{
Pranoko Rivandi ${ }^{1}$, Astuti Winda ${ }^{1, *}$, Dewanto Satrio $^{2}$, and Mahmud Iwan Solihin ${ }^{3}$ \\ ${ }^{1}$ Automotive and Robotics Program, Computer Engineering Department, BINUS ASO School of \\ Engineering, Bina Nusantara University, Jakarta, Indonesia 11480 \\ ${ }^{2}$ Computer Engineering Department, BINUS ASO School of Engineering, Bina Nusantara University, \\ Jl. K. H. Syahdan No. 9, Kemanggisan, Palmerah, Jakarta 11480, Indonesia \\ ${ }^{3}$ Faculty of Engineering, Technology and Built Environment, UCSI University, Cheras, Kuala \\ Lumpur 56000, Malaysia
}

\begin{abstract}
Automated vehicle security system plays an important rule in nowadays advance automotive technology. One of the methods which can be applied for a security system is based on biometric identification system. Fingerprint recognition is one of the biometric systems that can be applied to the security system. In this work, fingerprint recognition system to start the motorcycle engine is developed. The fingerprint of the owner and other authorized persons will be stored into the database, then while the time of starting the engine of the vehicle, the fingerprint will be validated with the database. The minutiae extraction method is applied to find the difference of fingerprint each other after turn the image into grayscale and thinning. After the extraction, the next step is finding the ridge edge and bifurcation. The result of the image will be used as input to the Artificial Neural Network (ANN) to recognize authorized person only. The experiment of fingerprint recognition system shows that automatic start-stop engine using fingerprint recognition system based minutiae extraction and Artificial Neural Network (ANN) has accuracy $100 \%$ and $100 \%$, respectively.
\end{abstract}

Keywords: Biometric recognition system, image processing, neural network.

\section{Introduction}

An Automated security system for vehicle plays an important role in the current advance automotive technology, which supported the industry revolution 4.0 [1]. One of the methods, which can be applied for security system, is based on biometric identification system [2]. The biometric recognition system is also an effective solution for personalizing vehicles, according to driver characteristics, allow drivers to access confidential information stored in vehicle databases and improving the security system of the vehicle [3]. Biometrics is automated methods used for person recognition based on physiological and behavioural characteristics [4]. The use of biometrics for identification or verification is increasing in because biometric qualities are extremely difficult to replicate, and do not change for a lifetime [5].

Fingerprint recognition is one of the biometric security systems that can be used [3]. Fingerprint recognition is one of many biometrics that commonly used to protect a system.

${ }^{*}$ Corresponding author: wastuti@,binus.edu 
Every single person has a different fingerprint pattern so that makes fingerprint biometric is very safe as a security system. Other than that, fingerprint recognition has a high accuracy. There is another biometrics system like voice recognition, face recognition, iris recognition, and palm vein recognition [6].

Figure 1 shows the percentage of all the biometrics often implements in the security system. The figure shows that the fingerprint recognition is the most favourable used for the security system. Each biometrics have an advantage and a disadvantage as shown in Table below. In Table 1, there is three comparison thing from all biometrics. Fingerprint has a high accuracy, medium level of easy to use, but low user acceptance because for some cases that the user's finger is too dirty or dry.

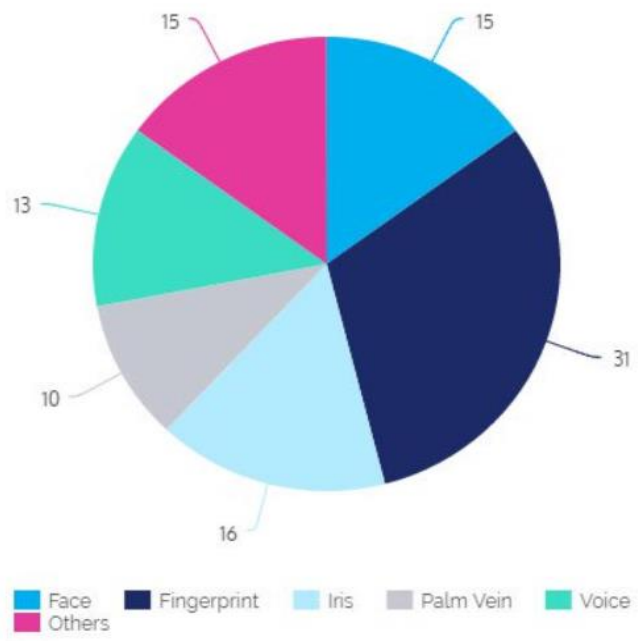

Fig. 1. Percentage of biometrics used [11].

Table 1. The difference of each biometrics [11].

\begin{tabular}{|c|c|c|c|}
\hline Biometrics & Accuracy & Easy to Use & User Acceptance \\
\hline Face & Low & High & High \\
\hline Fingerprint & High & Medium & Low \\
\hline Iris & High & Medium & Medium \\
\hline Palm Vein & High & High & Medium \\
\hline Voice & Medium & High & High \\
\hline
\end{tabular}

\section{Literature review}

There are three main steps on fingerprint recognition; they are image enhancement, image extraction, and image recognition [12]. The first step is Image enhancement, the function of this step is to make a better quality of fingerprint images as input for image extraction. The second important step is image extraction. In this function, the unique fingerprint pattern of each person is developed. The output of image extraction becomes the input for fingerprint recognition system. In the recognition system, the fingerprint will be matching a fingerprint with the fingerprint on the database. The output of the recognition system will use as input to the Arduino, which will be activated to start and stop the engine. From Arduino connected to the active relay to starting the engine if the fingerprint is authorized with the database. 
There are some steps on image enhancement of fingerprint, they are Histogram Equalization, Fingerprint Enhancement by Fourier Transfer, and Fingerprint Image Binarization [13]. After image enhancement is minutiae extraction. To get a unique pattern of a fingerprint, the image must be thinned before. Fingerprint recognition has some way of matching a fingerprint-like minutiae match and neural network. Hardware needed for this project is an Arduino, fingerprint sensor, and relay. So, below is the diagram of the basic flowchart of fingerprint recognition system on this project.

\subsection{Image enhancement}

Fingerprint image enhancement is used to make the image clearer for easy further operations. Since the fingerprint acquired from fingerprint sensor is not in the perfect quality, those enhancement methods, for increasing the contrast between ridges and valleys [14]. This method is also used for connecting the false broken points of ridges due to an insufficient amount of ink, are very useful for keeping a higher accuracy to fingerprint recognition $[15,16]$. There are three types of method for image enhancement; Histogram Equalization, in this type is done by expanding the pixel value distribution of an image so as to increase the perceptional information [13]. The seconds type of image enhancement is Fourier Transform, this method is done by connected some falsely broken points on ridges and to remove some false connections between ridges [15]. The last type of enhancement type is binarization, in this type basically stating the obvious, which is that the true information that could be extracted from a print is simply binary; ridges versus valleys [15].

\subsection{Minutiae extraction}

Before extraction, the image of fingerprint should be thinned first. Minutiae can be defined as the points where the ridge lines end or fork. So the minutiae points are the local ridge discontinuities and can be of many types. These types are [15]:

i. Ridge ending: a ridge that ends abruptly.

ii. Ridge bifurcation: a single ridge that divides into two ridges.

iii. Short ridge, island or independent ridge: a ridge that commences travelling a short distance and then ends.

iv. Ridge enclosures: a single ridge that bifurcates and reunites shortly afterwards to continue as a single ridge.

v. Spurs: a bifurcation with a short ridge branching off a longer ridge.

vi. Crossover or bridge: a short ridge that runs between two parallel ridges.

\subsection{Fingerprint recognition}

Fingerprint recognition is the process of comparing questioned and known fingerprint against another fingerprint to determine if the impressions are from the same finger. It includes two sub-domains, one is fingerprint verification and the other is fingerprint identification.

Fingerprint verification is to verify the authenticity of one person by his fingerprint. Fingerprint identification is to specify one person's identity by his fingerprint without knowledge of the person's identity, the fingerprint identification system tries to match his fingerprint with those in the whole fingerprint in the database. Artificial Neural Network (ANN) is applied as a method for fingerprint recognition. 


\subsubsection{Artificial neural network}

Artificial Neural networks (ANN) algorithm is used to simulate same or all the characteristic of the biological neurons that form the structural constituents of the brain. There are two important phases in ANN, training phase and testing phase. In the training phase, data train in ANN in order to develop the model of the data. Testing phase, the data are tested into the model that had been developed [18].

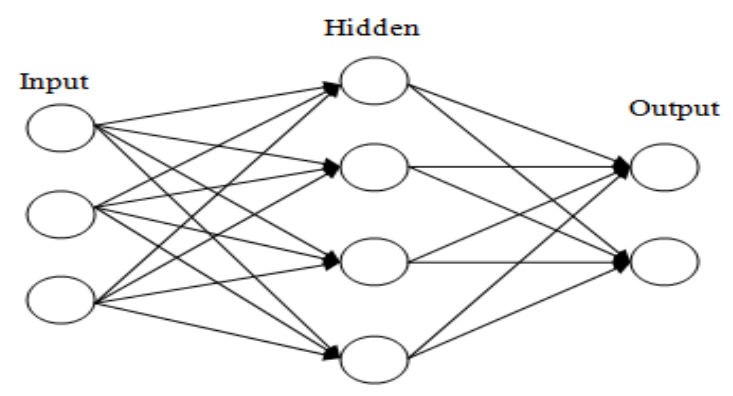

Fig. 2. MLP neural network.

From fingerprint sensor to take an image of a fingerprint which connected with Arduino. Software that used to support this project is MATLAB. Image enhancement, image extraction, and fingerprint recognition are using MATLAB.

\section{Research methodology}

In this project start-stop engine based on fingerprint recognition, considers Industrial Revolution 4.0 in which technological advances in automotive became the important focused are rapidly developing. The research methodology of this project refers to [21] with the approach through quantitative approaches.

A fingerprint taken from the sensor will be processed in the MATLAB through fingerprint recognition system. The output from the fingerprint recognition is used as input to Arduino to trigger the relay. The engine is starting if the output from fingerprint recognition is true or matched.

In order to achieve the objective of this project, the following procedures will be considered:

i. This project will start with the understanding of fingerprint image processing, artificial intelligence technique, and hardware development.

ii. Development of fingerprint detection, and feature extraction technique.

iii. Development of fingerprint recognition system.

iv. The development of the fingerprint recognition system is done in this step using ANN as an intelligent recognition system.

v. Development of Matlab for an automatic start-stop engine based on fingerprint system.

vi. Matlab® used as a tool to develop start-stop engine program.

vii. Development of hardware integrated system for an automatic start-stop engine based on fingerprint recognition system.

viii. Hardware used Arduino in order to get the input from Matlab and trigger the relay to activate the vehicle.

ix. Analysis of the performance of the proposed system. 


\section{Experimental work}

In this section, the performance of the proposed Automotive Start-Stop Engine Based on Fingerprint Recognition system. There are three important subsections, they are experimental setup, experimental result, and experimental analysis.

\subsection{Experimental design}

In this experiment, the data are taken from the real situation, with two persons and each of them took data of their finger ten times. Five of the finger data will be used as training data and five other data used as testing data.

Figure 3 that shows the fingerprint system scheme, the fingerprint sensor sent the data to the Arduino that will transfer to PC to be identified. The identify finger print will active the relay on the engine.
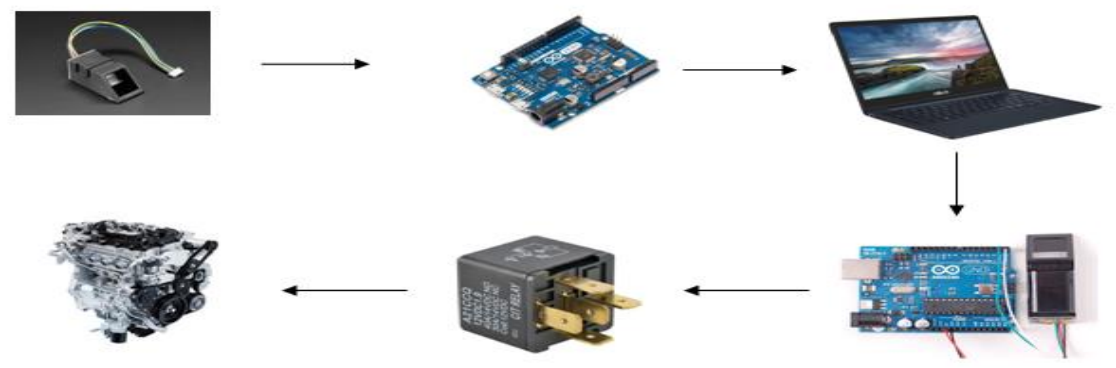

Fig. 3. Overall of the proposed system.

\subsection{Experimental results}

In order to evaluate the effectiveness of the proposed fingerprint recognition system, the experiment is then into different phases (training and testing phases). In the training, data of authorized person are recorded using fingerprint sensor. The data processed on a fingerprint recognition system using a MATLAB program until the system can find the unique pattern of someone's fingerprint. The pattern of authorized person then saved in the database, the fingerprint of authorized person will be cross-checked using Artificial Neural Network with the data in the database. The image scanned from the fingerprint sensor is a grayscale image as shown in Figure 4 below.

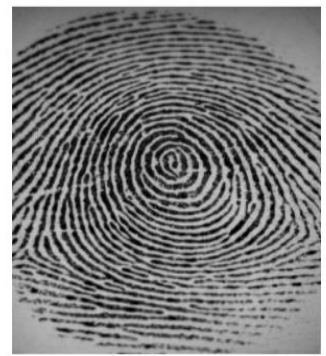

Fig. 4. Fingerprint scanned from a fingerprint sensor. 
From Figure 5 the image above should be converted to binary image in order to separate background and foreground of the image. Then the binary image is cropped within the core of fingerprint to reduce the area to be processed. In this process as in Figure 6.

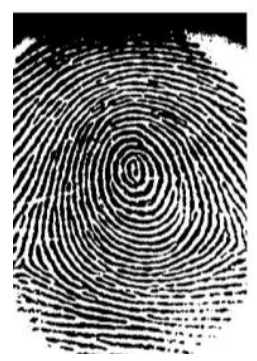

Fig. 5. Binary image.

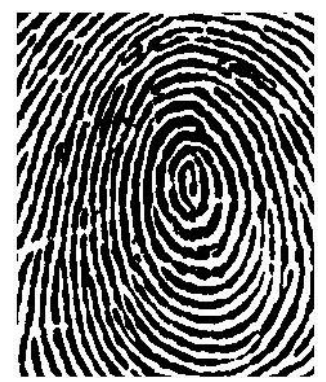

Fig. 6. Core of fingerprint.

After turning the image into a binary of core fingerprint, the next step is thinning image (Figure 7). Thinning is an operation to remove selected foreground pixels from binary images, so the image looks like a line of the fingerprint. The last operation of fingerprint recognition is minutiae extraction (Figure 8). With minutiae extraction, the system will find many types of fingerprint ridge as explained at 4.4 minutiae extraction. The image of minutiae extraction is shown below.

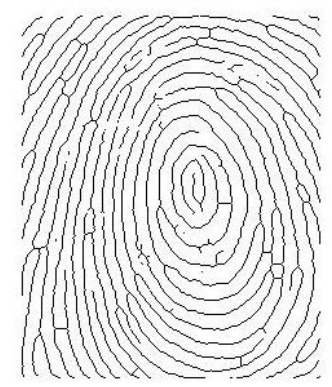

Fig. 7. Thinning image. 


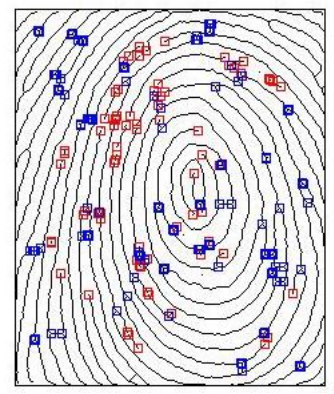

Fig. 8. Image of minutiae extraction

The result of the minutiae extraction technique is an extraction matrix which representation of the significant location of the bifurcation. Table 2 shows the output from the minutiae extraction will be used as input to the recognition system that applies neural network. The result of the experiment resulting $100 \%$ and $100 \%$ for both training and testing phase accuracy, respectively.

Table 2. Experiment result.

\begin{tabular}{|c|c|c|}
\hline Person & Training Accuracy (\%) & Testing Accuracy (\%) \\
\hline Person 1 & 100 & 100 \\
\hline Person 2 & 100 & 100 \\
\hline
\end{tabular}

\subsection{Experimental analysis}

In this project, Automatic Start-Stop Engine Based on Fingerprint Recognition is developed. The data are taken from fingerprint sensor. Then the fingerprint data processed in the fingerprint recognition using MATLAB. The result from MATLAB became an input to the Arduino and give a command to relay. The relay activates the engine. The result of this experiment has an accuracy of $100 \%$ in training phases and the accuracy of testing is $100 \%$. From the result can be concluded that the proposed system is relatively reliable since it has a good accuracy of $100 \%$ and $100 \%$ for training and accuracy phases respectively.

\section{Conclusion}

Safety and comfort as a part of human factor play important role in nowadays advance automotive technology. Security system on a vehicle, as one of the safety aspects, is susceptible to an unidentified person. The conventional security system has potential to be lost, stolen or duplicated by someone. Due to that reason, a biometric method can be one of the solutions for the authentication for the driver by using their fingerprint is one of the potential solutions. The experiment shows the system reliable to recognize the authorized person, since it has good training and testing accuracy, respectively. The face recognition system is then installed in Hardware which consists of Arduino ${ }^{\circledR}$, relay, and the engine, the result shows that the system is a success to be implemented. 


\section{References}

1. World economic Forum. ASEAN 4.0: What does the Fourth Industrial Revolution mean for regional economic integration? Switzerland : World Economic Forum (2017). p. 18. https://www.adb.org/sites/default/files/publication/379401/asean-fourth-industrialrevolution-rci.pdf

2. M. Godase, A.N. Barbole. IJCNWMC, 2,3:13-26(2012). http://www.tjprc.org/publishpapers/2-3-1346383900-2.Comp\%20Networking\%20\%20IJCNWMC\%20-\%20Biometric\%20-\%20Milind\%20G.pdf

3. N. Kiruthiga, L. Latha. IJARTET, 1,2:10-16(2014). https://www.researchgate.net/publication/318787447_A_Study of Biometric_Approac $\mathrm{h}$ for Vehicle Security System_Using Fingerprint Recognition

4. M.M.H. Ali, V.H. Mahale, P. Yannawar, A.T. Gaikwad. Overview of fingerprint recognition system. International Conference on Electrical, Electronics, and Optimization Techniques (Chennai, India, 2016). https://ieeexplore.ieee.org/document/7754900

5. A. Jain, P. Flynn, A.A. Ross. Handbook of Biometrics. Verlag, US: Springer (2008). p. 556. https://www.springer.com/gp/book/9780387710402

6. Priyanka. Fingerprint recognition techniques and its applications. International Conference on Advances in Engineering and Technology Research (Kanpur, India, 2014). p. 353-358. http://toc.proceedings.com/24683webtoc.pdf

7. D. Ibrahim. Microcontroller based applied digital control. England: John Wiley and Sons, Inc (2006). p. 311. https://onlinelibrary.wiley.com/doi/book/10.1002/0470863374

8. S.A. Kumar, C.K.A.A. Cholzaa, R. Arulraj, P.C. Kumar, R. Somasundaram. IJIRSET, 7,1:147-150(2018). http://www.ijirset.com/upload/2018/ncfcsps/J25 Biometric.pdf

9. R. Prashantkumar, V.C. Sagar, S. Santosh, S. Nambiar. IJESET, 6,3:324-334(2013). https://www.slideshare.net/PrashantkumarR1/6-n13-ijeset0603127v6iss3324334pdf

10. V.M. Ibrahim, A.A. Victor. IJERD, 2,10:18-22(2012). http://www.ijerd.com/paper/vol2-issue10/D02101822.pdf

11. Danny, T. Top five biometrics: Face, fingerprint, iris, palm and voice. [Online] from https://www.bayometric.com/biometrics-face-finger-iris-palm-voice/ (2017). [Accessed on 2 October, 2019].

12.J.C. Russ. The image processing handbook. Washington D.C.: CRC Press (2002). https://www.amazon.com/Image-Processing-Handbook-Fourth/dp/084931142X

13. A. Kadir, A. Susanto. Teori dan aplikasi pengolahan citra. [Theory and application of image processing] Yogyakarta: Andi (2013). p. 640. [in Bahasa Indonesia]. http://andipublisher.com/produk-0513004726-teori-dan-aplikasi-pengolahancitracd.html

14. D. Gunawan, F.H. Juwono. Pengolahan sinyal digital dengan pemograman matlab [Digital signal processing with matlab programming]. Yogyakarta: Graha Ilmu (2012). p. 266. [in Bahasa Indonesia]. http://library.uny.ac.id/sirkulasi/index.php?p=show detail\&id=52445

15.Z.S. Barham, A. Mousa. Fingerprint recognition using MATLAB graduation project. Palestine: An-Najah National University (2011). p. 74. https://www.academia.edu/4979773/Fingerprint_Recognition_using_MATLAB_Gradua tion project Acknowledgement

16. A. Saxena, S. Sharma, S. Gaur, S. Chauhan, Varshney. International Journal of Scientific Research and Management Studies, 2,1:66-71(2015). http://www.ijsrms.com/media/8I13-IJSRMS0201663-v2-i1-66-71.pdf 
17.W. Zafar, T. Ahmad, M. Hassan. Minutiae based fingerprint matching techniques. 17th IEEE International Multi Topic Conference: Collaborative and Sustainable Development of Technologies (Karachi, Pakistan, 2014). IEEE INMIC 2014Proceedings, p. 411-416. https://www.researchgate.net/publication/301403693_Minutiae based fingerprint_matc hing techniques

18. Koivo, H.N. (2008). Neural networks: Basics using MATLAB Neural Network Toolbox, 1-59. [Online] from http://staff.ttu.ee/ jmajak/Neural_networks basics_pdf (2008). [Accesed on 2 October, 2019].

19.J.M. Keller, D. Liu, D.B. Fogel. Fundamentals of computational intelligence: Neural networks, fuzzy systems, and evolutionary computation. New Jersey: Wiley IEEE Press (2016). p.77-100. http://www.amazon.com/Fundamentals-Computational-IntelligenceEvolutionary-Computation/dp/1119214343/

20. P. Liu, H. Li. Fuzzy neural network theory and application. In: Machine perception and artificial intelligence. H. Bunke, P.S. Wang (Eds.). Singapore: World Scientific (2004). p. $1-61$. https://pdfs.semanticscholar.org/63d3/a25562056e09cbe82170d80785f9a23d9793.pdf

21.J.W. Creswell. Research design: Qualitative, quantitative, and mixed methods approaches. London: Sage Publication (2017). p. 273.

https://www.amazon.com/Research-Design-Qualitative-QuantitativeApproaches/dp/1452226105 